\title{
IV Ancora sui collaboratori di Isacco Argiro: numerose mani simili
}

Il confronto tra la scrittura di Argiro e quella dell'Anonimo G condotto in II.2.1 mostra quanta attenzione richieda una corretta distinzione di queste mani. Non sorprende, dunque, che gli studiosi abbiano in alcuni casi attribuito il lavoro dell'uno alla mano dell'altro, o preferito sospendere il giudizio di fronte ad alcune attribuzioni. Somiglianze e affinità interessano anche altre mani: l'Anonimo $G$ non è che uno dei copisti con cui Argiro è stato confuso. Menziono solo i casi di attribuzione, a mio avviso, erronea riscontrati durante la presente ricerca: indagini successive arricchiranno di sicuro questa lista con esempi analoghi.

\section{IV.1 Anonimo G?}

È nota l'erronea identificazione nell'Almagesto Marc. gr. Z. 310 dell'Anonimo G, formulata da Bianconi e in seguito corretta da Brigitte Mondrain, che ne ha giustamente riferito la copia ad Argiro. ${ }^{165}$ Sembrano essere suscettibili di una nuova valutazione anche i ff. 62v, 1. 16-64v, 192r, 1. 16-197v, 226v, 1. 15-229v, 248v della raccolta storica Laur. Plut. 70.5: questi fogli, che Brigitte Mondrain ha attribuito ad Argiro, sono da ricondurre più verosimilmente alla produzione scrittoria dell'Anonimo G, come proposto in precedenza da Bianconi. ${ }^{166}$

A sostegno di questa attribuzione concorrono più elementi grafici. Anzitutto l'aspetto d'insieme, caratterizzato da una maggiore rigidità del tracciato e staticità delle forme rispetto a ciò che è proprio della scrittura di Argiro. Inoltre, nel Laurenziano sono rintracciabili alcuni ductus singulares ${ }^{167}$ distintivi della scrittura dell'Anonimo: beta maiuscolo con asta verticale mai debordante in basso oltre l'ideale rigo di base e con ansa inferiore talvolta ingrandita (f. 63v, 1. 10); csi nella forma destrogira con seconda ansa talora più sporgente verso l'esterno (f. 193r, 1. 19); zeta destrogiro, poco comune nella scrittura di Argiro (f. 196v, 1. 3); phi con corpo rotondo e non ovale (f. 194v, 1l. 8, 9). Si aggiungano alcune lettere in legatura, come la realizzazione del gruppo alpha-pi (f. 63v, 11. 6, 10, 11) in un legame tipico dell'Anonimo G, che Pérez

165 La falsa attribuzione del Marc. gr. Z. 310 all'Anonimo $G$ postulata in Bianconi (2003) 552, n. 123, è stata accolta in Pérez Martín (2008) 442; correzione in Mondrain (2007) [2008] 166 e n. 16.

166 Il codice, messo in relazione con Gregora già da Mazzucchi (1994) 210, e Canfora (1995) 234, è stato analizzato in seguito da Bianconi (2003) 552, n. 123, e da Mondrain (2007) [2008] 166, n. 16, e soprattutto da Clérigues (2007).

167 Cf. Bianconi (2012) 311, che trae la citazione da Montfaucon (1708) 316: «tachygraphi autem ii qui abbreviationibus multis, ductibusque singularibus atque expeditiore manu scripturam absolvunt». 
Martín ritiene «de aire chipriota» ${ }^{\mathbf{1 6 8}}$, e le multiformi possibilità in cui viene realizzata la legatura epsilon-csi, mai, tuttavia, in una delle combinazioni preferite da Argiro.

Tra i casi da riesaminare rientra anche l'identificazione, proposta da Polemis, ${ }^{169}$ della mano di Argiro nel Par. gr. 1246. Questo codice, testimone del Contra Palamam di Giovanni Ciparissiota, ${ }^{170}$ è composto da tre unità riferibili al medesimo ambiente di produzione. ${ }^{171}$ Alla sua realizzazione - diversamente da quanto sostiene Polemis che ha individuato una sola mano - si sono alternati due copisti: per l'appunto Argiro e l'Anonimo G. Quest'ultimo ha vergato il primo (1r-160r) e il terzo (ff. 283r-388v) blocco del codice, recanti rispettivamente i primi due libri e il quinto del trattato di Ciparissiota. ${ }^{172}$ Argiro è intervenuto nella seconda unità (ff. 161r-282v) dell'esemplare, trascrivendo i libri terzo e quarto.

Come già ricordato in precedenza, Polemis sostiene che la copia del codice sia opera dello stesso copista che ha vergato i codici Vatt. grr. 604, 1094, 1096, 1102, ma esprime riserve quanto all' identificarlo con Argiro, ${ }^{173}$ non tenendo conto delle ricerche compiute negli ultimi anni, ${ }^{174}$ che hanno definito una volta per tutte la personalità grafica di quest'ultimo. ${ }^{175}$

È dunque necessario un esame comparativo delle due mani all'opera nell'esemplare di Parigi. Le loro scritture, per quanto prossime per la comune aderenza allo

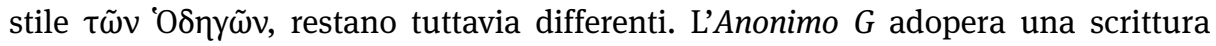
dal ductus posato, ad asse diritto e dal tracciato angoloso, con esecuzioni rigide e squadrate di beta maiuscolo, csi sinistrogiro nella forma tradizionale derivante dalla minuscola antica, e alpha-pi, nella cosiddetta forma "cipriota". Presento in un prospetto schematico le peculiarità della scrittura dell'Anonimo $G$ che ricorrono tanto nel Vat. gr. 1095, quanto nei blocchi liminali del codice di Parigi, che si devono pertanto riferire alla sua mano (Tabella IV):

168 Pérez Martín (2008) 440.

169 Polemis (2012) LV.

170 Sul Par. gr. 1246 e sul ruolo rivestito dall'esemplare nella tradizione manoscritta dell'opera di Ciparissiota rinvio a Gioffreda (2017).

171 La scheda è infra 214-218.

172 Sulla tradizione manoscritta del Contra Nilum Cabasilam si veda l'introduzione di Maragkudakis (1984) e Gioffreda (2017)

173 Polemis (2012) LV: «Mercati believed that the scribe might be Argyros himself [...] The fact that this hand has made, at two points, corrections to the text of the treatise of Argyros, as Mercati remarks, does not prove anything, since the scribe did not interfere with the text of the anonymous author in radical way, so that we might have been justified to identify the scribe with the author of it. In other words: even if that hand is identical with the hand of Argyros, as Mercati was inclined to believe, which is rather doubtful, nothing permits us to consider the text of Vatican's transcribed by it as its own product».

174 Già Rigo (2014) 289 n. 11, nota come Polemis non tenga conto dei risultati raggiunti dalle ricerche paleografiche.

175 Bianconi (2008) 359 e n. 70. 
Tabella IV:

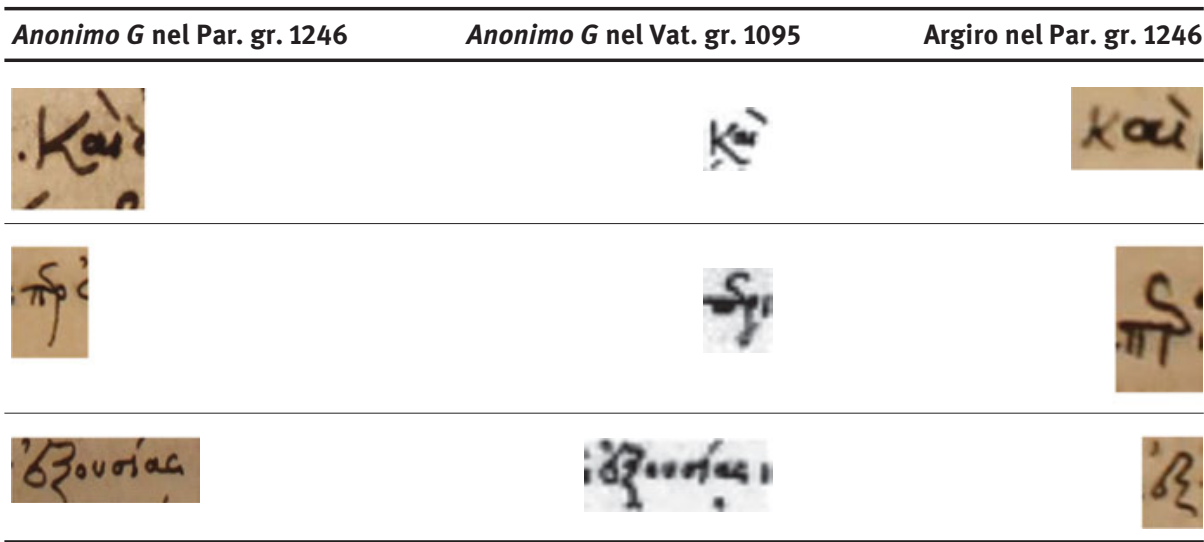

Argiro, che pure deriva dallo stile $\tau \tilde{\omega} v^{\circ} \mathrm{O} \delta \eta \gamma \tilde{\omega} v$ l'allungamento dei tratti diritti e obliqui di lettere, concede ampio spazio a forme dal disegno fluido, a tratti esuberanti, come in kappa e sigma lunato, così da presentare una catena grafica ordinata, in cui interagiscono senza contrasto elementi dal tracciato differente. L'impressione è quella di una scrittura vergata con un ductus posato, da una mano attenta e precisa, che si muove sulla pagina con esperienza e spontaneità. Una comparazione delle medesime esecuzioni nell’Aristotele Neap. III D 37 e nel Ciparissiota Par. gr. 1246 si rivela risolutiva ai fini attributivi (Tabella $\mathrm{V}$ ):

Tabella V:

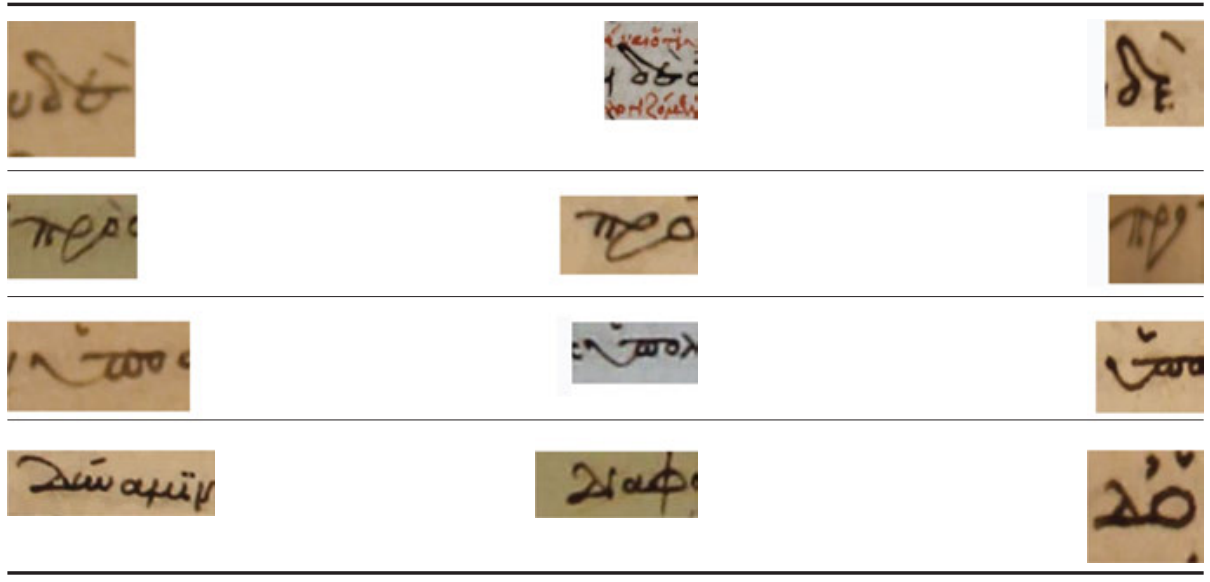


La correzione della reductio ad unum operata da Polemis è di un certo peso: la collaborazione fra Argiro e l'Anonimo G nel Par. gr. 1246 permette di ancorare ad un terreno più solido il più volte postulato rapporto di dipendenza grafica tra i due copisti. Prima d'ora, in effetti, le due personalità, le cui mani mostrano un'innegabile affinità, erano state accostate in virtù del legame con Gregora - per il quale l'Anonimo $G$ ha vergato numerosi manoscritti e di cui Argiro si dice esplicitamente allievo - e per il comune impegno nella trascrizione di opere astronomiche e matematiche.

A partire da questa attribuzione è facile poter riferire all'Anonimo $G$ anche i ff. 99r-194r della miscellanea antipalamitica Marc. gr. Z. $155 .{ }^{176}$ Essi, per riprendere le parole di Bianconi, sono stati vergati «in una grafia assai affine allo stile $\tau \tilde{\omega} v v^{\text {'O } \delta} \eta \gamma \tilde{\omega} v$ e, dunque, in una certa maniera, a quella dello stesso Argiro» ${ }^{177}$. Questi è d'altronde presente nei ff. 195r-198r della miscellanea, costituenti un'unità distinta dalla precedente, anche se ascrivibile al medesimo ambiente. Nei ff. 99r-194r spiccano, invece, tutti gli aspetti più connotanti della scrittura dell'Anonimo G: ductus posato, tracciato rigido e angoloso, aspetto calligrafico, oltremodo misurato; le forme tipiche di beta maiuscolo, di epsilon ingrandito pronto a legare con tratto mediano, di csi sinistrogiro in una forma tradizionale propria della minuscola antica, della legatura alpha-pi, nella forma "cipriota". Anche l'apparato decorativo, i titoli dei paragrafi e le lettere in ekthesis vergate in inchiostro rosso, la tipologia delle fasce decorative poste ad apertura dei testi, ritornano tanto nelle pagine del Marciano quanto in quelle del Parigino qui assegnate. ${ }^{178}$

Altro caso degno di nota riguarda il mancato riconoscimento di Argiro nel copista B del Par. gr. 1672. ${ }^{179}$ Il codice consta di due unità: quella originaria, formata dai ff. 1r-936v, tramanda la recensione planudea dei Moralia di Plutarco; quella posteriore, costituita dai ff. 937r-944v, conserva vari estratti da Appiano. ${ }^{180}$ Nella parte originaria, già Alexander Turyn distingueva tre mani: A (ff. 2r-706v, 870r-875v), B (ff. 707r-869v) e C (ff. 876r-936v), e proponeva di identificare B con lo scriba dei codici Ferrara, Ariostea II 178, Neap. III C 19, Par. gr. 2711, ovvero con l'Anonimo $G .{ }^{181}$ Mario Manfredini ha corretto la proposta di Turyn e ha giustamente riconosciuto l'Anonimo $G$ nel copista A del codice, identificando inoltre il copista C con Manuele Tzicandile. ${ }^{182}$ Nel copista

176 Questi fogli recano il De essentia et operatione di Demetrio Cidone; i ff. 195r-198r di mano di Argiro un florilegio di excerpta tratti da più autori e opere. Una descrizione dell'esemplare, infra 240-247. 177 Così in Bianconi (2008) 365; la somiglianza tra la grafia dell'Anonimo G e quella di Argiro è messa in rilievo anche da Mondrain (2007) [2008] 168. Per contro, la mano dell'Anonimo $G$ nei ff. 99r-194r è qui identificata per la prima volta.

178 Per la decorazione dei codici si rinvia alle relative schede, infra 240-247 e 214-218.

179 Per il codice, oltre alla scheda infra 226-232, rinvio a Martinelli Tempesta (2006) 72-75.

180 Questa seconda unità è stata copiata intorno alla metà del XV secolo da Giorgio Disypatos Galesiota, cui si deve anche il pinax al f. 1r: cf. RGK III, n. 99, con bibliografia.

181 Turyn (1972) I, 185.

182 Manfredini (1988) 125, e Id. (1989) 129-130. L'identificazione è stata confermata da Bianconi (2003) 553. 
B del Par. gr. 1672 si deve invece riconoscere Argiro. Anche in questo caso la sua scrittura si distingue da quella dell'Anonimo $G$ per una maggiore fluidità delle forme e per tratteggi caratterizzanti: delta maiuscolo ingrandito; beta maiuscolo con asta debordante; zeta in due varianti, quella stilizzata $\tau \tilde{\omega} v^{\circ} 0 \delta \eta \gamma \tilde{\omega} v$ e quella a forma di 2; kappa maiuscolo e phi con occhiello ovale.

Il codice di Parigi fu approntato prima del 1362, anno in cui Manuele Tzicandile completò una sua copia nell'attuale Bodl. Canon. gr. 93 + Ambr. D 538 inf. ${ }^{183}$ Il codice di Parigi conferma che Argiro e l'Anonimo $G$ lavorarono gomito a gomito. La loro collaborazione si colloca nella prima delle quattro fasi di interesse in cui ho suddiviso la produzione libraria di Argiro: quella degli anni '50 del XIV secolo. ${ }^{184}$

\section{IV.2 Anonimo A}

In questa valutazione delle mani simili, un'altra personalità grafica da considerare è quella dell'Anonimo $A$, con cui Argiro realizzò, come abbiamo visto nella sezione III.2.3, i quattro libri filosofico-astronomici Marc. gr. Z. 308, Neap. III D 37, Laur. Plut. 89 sup. 48, Scorial. Y.III.21.

La mano di Argiro e quella dell'Anonimo A sono state correttamente distinte nei ff. 1-34 del Marc. gr. Z. 308 con i Caelestia di Cleomede (ff. 1r-8v assegnati al collaboratore e quelli restanti ad Argiro), e nella copia dell'Euclide nello Scorial. Y.III.21 (ff. 105r-112v). La compresenza dei due scribi negli altri due codici necessita di precisazioni. ${ }^{185} \mathrm{Il}$ merito di aver riconosciuto Argiro nel copista principale dell'Organon aristotelico Neap. III D 37 spetta a Bianconi, che ha individuato l'Anonimo A nei soli ff. 38r-43r e ne ha proposto il riconoscimento nei ff. 1r-8v del Marc. gr. Z. 308. ${ }^{186}$ Che si tratti della stessa mano è provato da un breve confronto tra le due grafie: stesso tracciato sinuoso e disinvolto, stessi richiami talora più evidenti al Metochistesstil, tratteggio di lettere come zeta a forma di 2 che prosegue oltre il rigo di base, csi destrogiro, tau alto e phi con occhiello rotondo. L' Anonimo $A$ è però intervenuto anche in altri fogli dell'Aristotele di Napoli: alla sua mano e non a quella di Argiro dovranno essere attribuiti i ff. 5r-26v e i ff. 28r-29r, e, a partire dal f. $5 \mathrm{r}$ fino almeno al f. 23v, parte degli scolii. ${ }^{187} \mathrm{~A}$ indurre l'occhio in errore è senz'altro l'affinità grafica esibita dalle

183 Manfredini (1989) 130-131.

184 Si veda meglio infra $119-127$.

185 Bianconi (2008) 357, e sui codici si vedano le schede 190-193 e 185-189.

186 Sul codice di Napoli Bianconi (2008) 359 e n. 68 afferma: «i foll. 38r-43r (testo per intero, scolii fino alla 1. $38 \mu \varepsilon \tau \alpha \theta \varepsilon \dot{\sigma \varepsilon} \omega \varsigma)$ sono stati copiati da una mano molto simile, se non identica, a quella che ha copiato i foll. 1r-8v del Marc. gr. Z. 308 (coll. 636). Argiro vi è comunque intervenuto per locupletare gli scolii». Nel recente catalogo Formentin (2015) 168, ha accolto e recepito le identificazioni formulate da Bianconi, senza modifica.

187 Argiro è intervenuto anche in questi fogli per completare gli scolii; alla sua mano si devono ad esempio quelli nel marg. inf. del f. 5r, nel marg. inf. e interno del f. 11r, quelli del f. 16r e 16v. 
due mani: man mano che la collaborazione si fa più stretta, essa diviene stringente come nel Laur. Plut. 89 sup. 48 , dove il cambio di mano è tutt'altro che perspicuo. ${ }^{188}$ Nei fogli del codice di Napoli che ho attributo all'Anonimo A, egli fa uso di un scrittura estremamente chiara e ordinata, in cui si rileva un'inclinazione più pronunciata che nel Marciano per un tracciato spezzato e "geometrico" delle forme e per alcune soluzioni che denunciano una ripresa consapevole dalla scrittura di Argiro, dovuta al peso che nella formazione dei copisti/eruditi può aver svolto unicamente una relazione tra maestro e allievo. ${ }^{189}$

Nella tensione a caricare sulla propria scrittura gli stilemi del maestro, ideatore del codice aristotelico, avrà senza dubbio giocato un ruolo non secondario la tipologia del libro. Non stupisce che nel Marciano, un libro di lavoro, modesto per dimensioni e fattezze, la scrittura dell'Anonimo A poco si sia lasciata condizionare da quella di Argiro e abbia conservato movenze più vicine al Metochitesstil. Si notino il tracciato sinuoso e arrotondato delle lettere con occhiello, come alpha e phi, e le particolari soluzioni in legatura adottate per alcuni gruppi, tra gli altri quelle per alpha-lambda e per sigma-theta-alpha-iota. Tutte le peculiarità dell'Anonimo A, benché in una scrittura più controllata, permangono nei fogli del codice napoletano. Ritornano epsilon di forma recente inclinata a sinistra, zeta a forma di 2 prolungato in basso oltre il rigo di base, rho alto sul rigo, specie in legatura con lettera seguente, tau a forma di 7

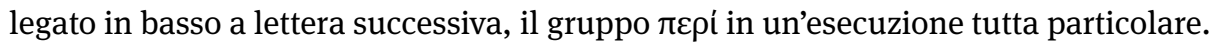
Questi indizi, decisivi già solo in luogo di attribuzione, fanno sentire maggiormente il loro peso qualora, come nel caso che segue, ci si trovi costretti a meglio specificare un'attribuzione precedentemente formulata.

Di non semplice definizione è in effetti la collaborazione tra Argiro e l'Anonimo $A$ nel Laur. Plut. 89 sup. 48, un importante testimone dell'Almagesto. ${ }^{190}$ Il codice è costituito da tre unità differenti: la seconda e più antica (ff. 7-169) è da riferire al pieno XIV secolo; la prima (ff. 1-6) e la terza (ff. 170-193) sono state prodotte nel XV secolo nel medesimo ambiente, come suggerisce la presenza dello stesso copista nelle due unità. ${ }^{191}$ L'unità più antica del codice (ff. 7-168) reca l'Almagesto di Tolomeo (ff. 20r$168 \mathrm{v})$ preceduto da una recensione bizantina degli anonimi Prolegomena ad Almagestum (ff. 7r-19r) approntata, secondo un'ipotesi recente, dallo stesso Argiro. ${ }^{192}$ Questo

188 Il problema dell'affinità grafica tra scribi è segnalato anche in Mondrain (2008) 126: «il est de fait notable que la distinction des mains et l'identification certaine de celle de notre scribe ne sont pas toujours aisées: c'est là un phénomène dont on peut parfaitement mesurer le poids lorsque l'on confronte l'écriture d'un maître et celle des ses disciples».

189 Rimando supra 29 e n. 128 la bibliografia citata.

190 Bianconi apud Acerbi (2013) 141, n. 50, ha preliminarmente ricondotto la copia del codice a Argiro.

191 Attualmente l'unica descrizione del codice è nel catalogo Bandini (1770) coll. 412-414; per una disamina più dettagliata si rimanda alla scheda infra 185-189.

192 Sui rapporti tra la versione della recensione tramandata in questo codice e la tradizione manoscritta restante si veda Acerbi (2013) 141, n. 50, in cui si avanza l'ipotesi che Argiro possa essere 
nucleo originario è stato in seguito arricchito con l'inserimento, all'inizio e alla fine, di due unità contenenti opere legate per contenuto o per tradizione al trattato tolemaico: i De eclipsis opuscula I e II e la Logistica di Barlaam Calabro. ${ }^{193}$

Una considerazione a sé merita il copista impegnato nella trascrizione della prima sezione e presente anche nella terza. La sua scrittura si distingue per eleganza e morbidezza dei tratti; la predilezione per le forme dal tracciato curvilineo consente di accostarla al gruppo delle scritture afferenti al cosiddetto Eugenikos-Schrift. ${ }^{194}$ A questa mano si deve il testo principale vergato nella prima unità del Laurenziano, ma anche le iniziali minori e i titoli vergati in inchiostro rosso nella terza unità del codice, così come alcune annotazioni marginali. Il suo interesse per le opere di carattere scientifico-filosofico trova conferma nella copia del Vat. gr. 1411, una miscellanea aritmetica e astronomica riferita all'ultimo quarto del secolo XIV. ${ }^{195}$ Sempre alla sua mano sembrano doversi riferire anche alcuni estratti presenti in diversi fogli del Vat. Urb. gr. 125, come ad esempio quelli presenti ai ff. $1 \mathrm{r},{ }^{196}$ 3rv, 306v, 1l. 26-33, e 307rv, fino alla 1. 17, e l'unico in inchiostro nero al foglio 308v; vi figurano citazioni di scritti platonici e passi tratti dagli oracoli caldaici. ${ }^{197}$

Ritorniamo alla parte più antica del Laur. Plut. 89 sup. 48, la cui trascrizione è frutto della collaborazione tra Argiro e l'Anonimo A. Ad Argiro si devono infatti riferire unicamente i ff. $7 \mathrm{r}-17 \mathrm{v}, 26$ e 136v, 1.5 a. i.-168r del Laurenziano, mentre la parte centrale del secondo blocco, ovvero i ff. $18 \mathrm{r}-136 \mathrm{v}$, è opera di questo suo allievo. ${ }^{198}$ In

l'autore di questa recensione, simile a quella del Marc. gr. Z. 310, ma rispetto a quest'ultima con notevoli novità.

193 Il primo copista ha aggiunto nella prima unità un'anonima Constructio astrolabii e i De eclipsis opuscula I e II (ed. Mogenet/Tihon/Donnet (1977)); il copista D ha vergato nei ff. 172-193 la Logistica (ed. Carelos (1996)). Il Laurenziano, come si evince dalla legatura decorata con ferri ad impressione, suggerisce un passaggio nell'officina del monastero del Prodromo di Petra, sul quale cf. Cataldi Palau (2008) I, 235-280.

194 La denominazione di Eugenikos-Schrift è stata coniata da Harlfinger (1977) 335 in relazione alla scrittura di Giovanni Eugenico, diacono e nomophylax attivo a Costantinopoli nel XV secolo e fratello di Marco Eugenico, sul quale oltre a RGK II, nr. 217, e PLP nr. 6189, si veda Petrides (1910).

195 Per una breve descrizione dell'esemplare Vaticano si vedano Mercati (1937) e Gautier (1988) 89-90, in cui la datazione del codice si deve a Paul Canart, oltre che Acerbi (2016). Su questo manoscritto, che va ricongiunto con i primi quattro fascicoli dell'Ambr. A 92 sup., ho in preparazione un contributo in collaborazione con Fabio Acerbi.

196 Ad eccezione di due note vergate al centro e al margine esterno del primo foglio di mano di Giovanni Eugenico (per l'identificazione di G. Eugenico nell'esemplare cfr. Fonkič (1979) 162 e n. 31). 197 A partire dall'attribuzione di questi fogli a Giovanni Eugenico avanzata dubitativamente in Fonkič (1979) 162 e poi confermata in De Gregorio (2000a) 344, n. 84 e nuovamente in De Gregorio (2014) 179, n. 2 e 186 n. 25, avevo proposto, apud Acerbi (2016) 138-143, di riconoscere anche nel Laurenziano e nel Vat. gr. 1411 la mano del famoso nomophylax antiunionista. Tuttavia, a seguito della correzione di questa mia identificazione ad opera di Giacomelli/Speranzi (2019), ho effettuato un riesame dei testimoni e del relativo materiale bibliografico e preferisco (ri)portare il copista del Laurenziano all'anonimato.

198 Gli scoli si devono ad Argiro anche nei fogli vergati dall'Anonimo A. 
questi fogli la svolta verso le scritture eleganti e ordinate legate al $\tau \tilde{\omega} v^{\circ} O \delta \eta \gamma \tilde{\omega} v$, di cui si colgono i primi accenni nelle pagine del Napoletano, sembra essere giunta a compimento, cosicché riesce difficile riconoscere la mano del collaboratore e distinguerla da quella di Argiro. Vergata con un ductus leggermente corsivo, che influisce appena sull'inclinazione della grafia - palese nella punta del gamma minuscolo e nella legatura a staffa di rho con lettera seguente - la mano di Argiro si presenta in una versione ordinata e altamente leggibile, nonostante il modulo piccolo della catena grafica. Al suo interno spiccano elementi specifici: beta maiuscolo con asta allungata al di sotto del rigo di scrittura; delta maiuscolo ad inizio rigo con tratto discendente debordante al di sopra del punto di intersezione; zeta a forma di 2; le molteplici realizzazioni di epsilon in legame con $c s i$, a ponte con pi e ridotto al solo elemento superiore in unione con ny, rho e phi.

La volontà di palesare un aspetto composto costringe l'Anonimo $A$ a rinunciare nei fogli del Laurenziano alle forme esuberanti e lo spinge da un lato ad adottare

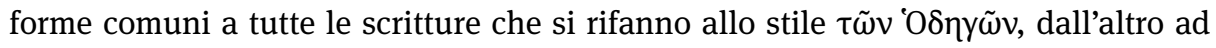
emulare in toto la scrittura del maestro. Resta però possibile, soprattutto nei primi fogli, in cui la tendenza emulativa è ancora all'inizio, riconoscerne la mano. La scrittura dal tracciato sinuoso presenta un leggera inclinazione verso destra, visibile nelle aste di alcune lettere, come quella del tau a forma di 7. L'andamento morbido delle forme denuncia un'antica aderenza al Metochitesstil, come pure gli occhielli di forma arrotondata di alpha, omicron e phi, caratteristiche che, tuttavia, si riducono coll'avanzare della copia e del processo di assimilazione imitativa. Quando il lavoro di trascrizione è ancora agli inizi, come ad esempio nei fogli 20r-21v, il copista ricorre più spesso che nelle pagine successive ad alcune delle sue forme precipue, quali beta con pance arrotondate, zeta a forma di 2 sporgente oltre il rigo di base, le legature alphacsi e epsilon-csi sempre uguali e le esecuzioni assai particolari dei gruppi di lettere

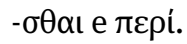

Segnalo a scopo esemplificativo alcune forme distintive proprie della mano dell' Anonimo A, così come presenti nel Marc. gr. Z. 308, nel Neap. III D 37 e nel Laur. Plut. 89 sup. 48. Esse risultano indispensabili per assegnare alla sua mano anche i ff. 105r-112v dello Scorial. Y.III.21 (Tabella VI).

Riassumo un processo di cui è stato possibile ricostruire alcune tappe: partendo da una scrittura molto vicina per aspetto e movenze al Metochitesstil, questo allievo di Argiro ne assimila la scrittura fino a pervenire allo stato finale, il Laur. Plut. 89 sup. 48. Qui la scrittura si è modificata a tal punto da poter essere ascritta al filone stilistico diametralmente opposto a quello di partenza: quello inaugurato dall'Anonimo $G$, in cui rientrano le scritture eleganti e ordinate di chiara derivazione dal $\tau \tilde{\omega} v$

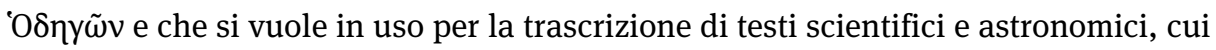
esse conferiscono una solennità, almeno esteriore, che è propria dei codici di contenuto sacro. 
Tabella VI:

Marc. gr. Z. $308 \quad$ Neap. III D $37 \quad$ Laur. Plut. 89 sup. 48

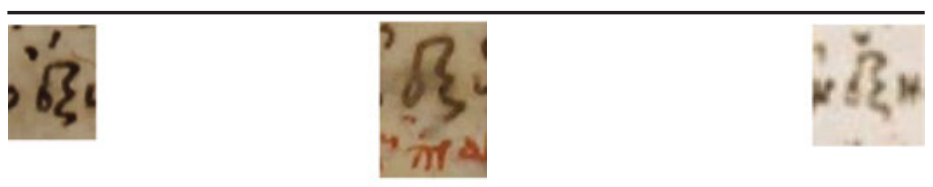
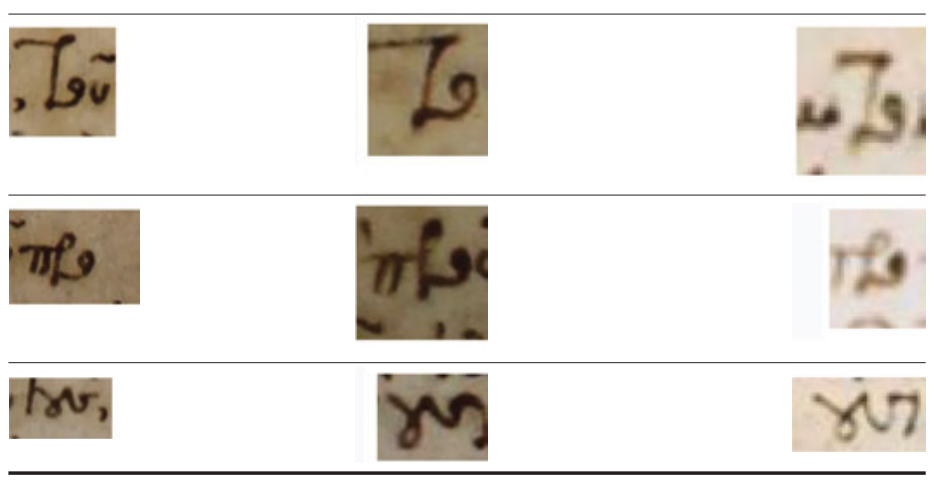

\section{IV.3 Anonimo B}

Un'ulteriore conferma del dilagare di questa moda e di un suo radicamento nella produzione di manoscritti di contenuto scientifico viene da un'altra testimonianza già ricordata: il composito Marc. gr. Z. 323, ${ }^{199}$ un'articolata e corposa miscellanea di testi astronomico-matematici formata dall'alternarsi di sezioni più antiche, risalenti al XIV secolo, e sezioni più recenti, riferibili al secolo XV, inserite tra le precedenti nel tentativo di completarle. ${ }^{200}$ Le unità più antiche si devono alla collaborazione tra Argiro e un altro scriba, denominato (si veda la sezione III.2.4) Anonimo B. Quest'ultimo fa uso di una scrittura molto simile a quella di Argiro e trae elementi ora dal suo registro corsivo, ora da quello posato; presenta ductus corsivo, evidente nell'inclinazione a destra, tracciato leggermente angoloso, frequenti abbreviazioni e segni tachigrafici,

199 Anche in questo caso l'individuazione della mano di Argiro nella miscellanea astronomica si deve a Bianconi (2008) 358, e Mondrain (2007) [2008] 167. Sulla struttura della miscellanea, formata dall'alternarsi di unità più antiche (II, IV, VI) e unità più recenti (I, III, V, VII) si veda la scheda infra 257-264. $200 \mathrm{Al}$ copista delle sezioni (I, III, V, VII) riferibili ai primi decenni del XV secolo (ff. 1r-22v, 25r-37v, 41r-70r, 71r-169v, 211r-212r, 214rv, 222r-225v, 226r-244v, 245r-249v, 258r-263r, 479r-487r, 487v), si devono anche i Vatt. grr. 573 (ff. 51r-214v); 792 (ff. 1r-24v e 354r-360v); 1058 (ff. 2r, 1. 2 ab imo-3r, 4r-8v, 9r-260v); 1709 (ff. 1r-196v, 203r-210v) (cf. in parte Mercati (1926) 71, n. 5); Marc. gr. Z. 335 (coll. 645), Par. gr. 2494 (ff. 122r-123r) e Vind. suppl. gr. 75 (ff. 29r-49v). Su questo copista, denominato $A G$, ho in preparazione un lavoro con Fabio Acerbi. 
ma resta nel complesso leggibile e composta. ${ }^{201}$ Circa la suddivisione del lavoro tra Argiro e questo scriba del Marc. gr. Z. 323, pur condividendo quanto definito da Bianconi e Mondrain, me ne discosto marginalmente col riferire il f. 220r-220v, 1. 16, alla mano dell'anonimo anziché a quella di Argiro. Dirimenti in questa scelta mi sembrano alcuni tratteggi specifici, come beta maiuscolo con asta poco sporgente in basso, zeta destrogiro dal tracciato sinuoso, theta completamente aperto a destra e la forma di csi in legatura con epsilon. Questi tratteggi sono riassunti nella tabella seguente, dove sono messi a confronto con le varianti delle stesse lettere nella scrittura di Argiro (Tabella VII):

\section{Tabella VII:}

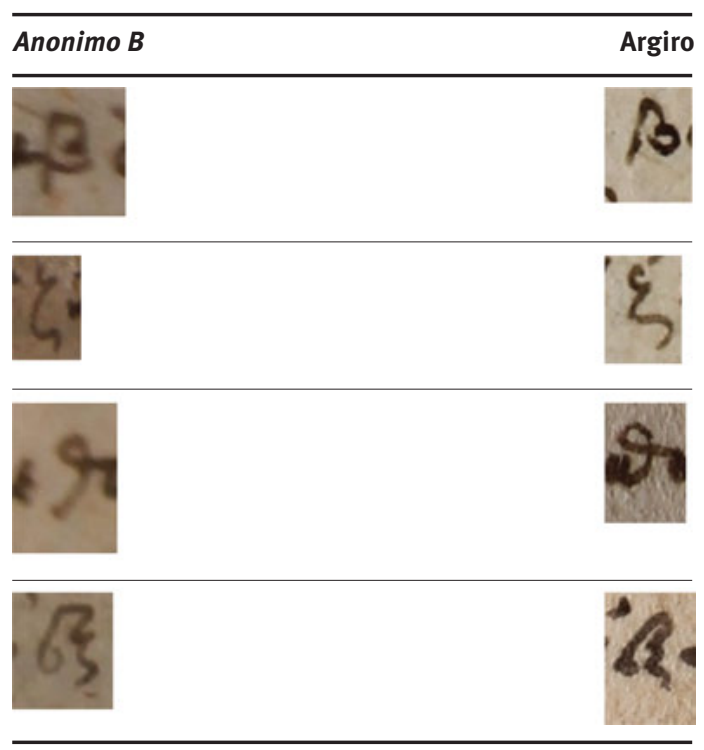

Anche l'Anonimo $B$ ha preso parte alla trascrizione di opere scientifiche: il Quod optimum initium cyclorum di Argiro nel Vat. gr. 573 (ff. 11r-14v) e il De usu astrolabii di Niceforo Gregora nello Scorial. Y.III.21 (ff. 90r-93v). Alla sua mano, tuttavia, piuttosto che a quella dell'Anonimo $G$ mi sembra si debbano assegnare i ff. 23r-32v del Vat. gr. 1086 con la Oratio in S. Theophanem di Niceforo Gregora. ${ }^{202}$ L'aspetto d'insieme

201 Nelle sezioni risalenti al secolo XIV del codice, ovvero II, IV, VI si sono alternati Argiro (ff. 210v, 215r-215v, l. 18, 220v, 1.17-221v, 1. 4, 285r, 1. 14-288v, 1. 16, 394r-398v, 1. 13, 400r, 468rv, 471r-476r, 1. 19) e l' Anonimo B (ff. 171r-204r, 205r-207r, 1. 25, 208r-210r, 217v-218v, 266r-285r, 1. 12, 289r-303v, 304r-305r, 1l. 1-6, 305v-308r, 309r-343r, 1. 16, 343v-382v, 384r-393r, 1. 25, 403r-467v, 469r-470v, 1. 8), meglio infra 257-264.

202 Cf. BHG 1795. 
è armonioso, il disegno meno rigido e si riscontrano tratteggi tipici della sua mano: delta minuscolo con ansa arrotondata, csi destrogiro sinuoso, omega aperto, legatura di alpha-csi ed epsilon-csi stondate. 